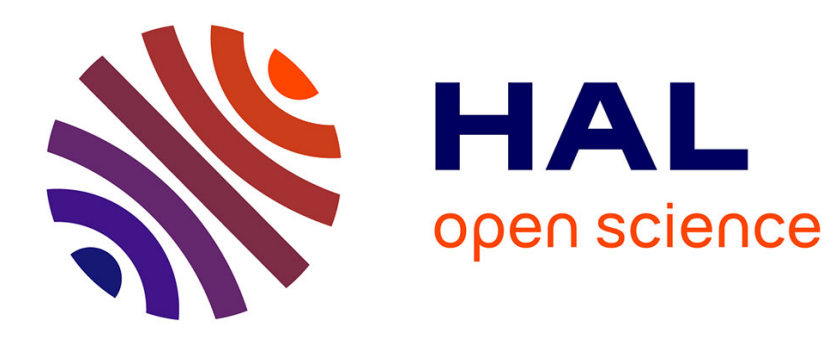

\title{
Web et bibliothèques, entre métaphore et mimèsis
}

Bruno Menon

\section{To cite this version:}

Bruno Menon. Web et bibliothèques, entre métaphore et mimèsis. Journées d'étude du groupe TICIS

- SFSIC : Le web a-t-il un sens?, Dec 2010, Paris, France. halshs-00647868

\section{HAL Id: halshs-00647868 \\ https://shs.hal.science/halshs-00647868}

Submitted on 2 Dec 2011

HAL is a multi-disciplinary open access archive for the deposit and dissemination of scientific research documents, whether they are published or not. The documents may come from teaching and research institutions in France or abroad, or from public or private research centers.
L'archive ouverte pluridisciplinaire HAL, est destinée au dépôt et à la diffusion de documents scientifiques de niveau recherche, publiés ou non, émanant des établissements d'enseignement et de recherche français ou étrangers, des laboratoires publics ou privés. 


\section{Web et bibliothèques, entre métaphore et mimèsis}

\section{Bruno Menon}

\section{Laboratoire Paragraphe - Université Paris 8}

\section{Résumé}

Depuis l'avènement de la Toile, métaphores et comparaisons rapprochant le Web et les bibliothèques sont récurrentes et insistantes; nombre de réflexions et d'écrits font état d'analogies et d'homologies entre ces deux dispositifs, jusqu'à justifier des pratiques mimétiques, parfois croisées, et de troublantes mises en abyme. De tels rapprochements peuvent étonner, tant les deux dispositifs sont dissemblables. C'est pourquoi il ne semble pas inutile d'en entamer un inventaire et de tenter d'en clarifier les facteurs. Quatre figures, de ces rapprochements sont évoquées :

1. I'utopie originelle du Web comme bibliothèque, et l'image d'une bibliothèque dysfonctionnelle ;

2. le Web catalogué ou incorporé dans les bibliothèques, et, en miroir, les avatars des bibliothèques sur le Web, modèle de bibliothèque universelle ;

3. le fantasme d'une bibliothèque mimant le Web (2.0) ;

4. le projet d'un Web sémantique structuré comme une bibliothèque. 


\section{Un aspect de l'imaginaire du Web}

Le Web a vingt ans ${ }^{1}$ (ce qui peut s'écrire " 2.0 »). A l'heure du Web socio-communicationnel, une archéologie du Web 1.0, ou Web informationnel / documentaire serait-elle de mise, même s'il est loin d'avoir disparu ? Au reste, les traces lisibles qui nous en sont offertes cohabitent et s'entremêlent en différentes strates et la temporalité n'y est guère décisive. Les pistes de lecture de ces traces, ou faciès, sont bien entendu multiples: technologiques, sociologiques, culturelles... Ce que nous tentons d'explorer ici est de l'ordre de l'imaginaire et du symbolique, dimensions bien peu présentes dans les Internet Studies.

C'est à travers le recueil et l'examen d'instances d'une métaphore massivement récurrente, celle de la bibliothèque, que nous aborderons l'un des aspects de l'imaginaire du Web, tout en évoquant "la possibilité que le discours métaphorique dise quelque chose sur la réalité " [Ricœur, 1975, p. 10]. Entre Web et bibliothèques, imbrications, mises en abyme et mises en relation spéculaires dessinent d'autres figures en lesquelles semble-t-il cette métaphore initiale se prolonge. Elles sont ici regroupées, faute de mieux, sous le vocable de mimèsis, au prix d'une distension de cette notion (ou pas : " chez Platon, [le concept de mimèsis] reçoit une extension sans borne ; il s'applique à tous les arts, aux discours, aux institutions, aux choses naturelles qui sont des imitations des modèles idéaux, et ainsi aux principes mêmes des choses "[Ricœur, 1975, p. 54]).

A la fois observateur de cette mimèsis et usager des dispositifs qui en sont les parties prenantes, nous avons tenté de n'utiliser pour cette étude que les ressources du Web, cherchant à vérifier ainsi cette prédiction faite aux débuts des bibliothèques numériques : "Within a few years, I suggest ten years, the on-line library will be the standard research library in most disciplines" (Dans quelques années, dix dirais-je, la bibliothèque en ligne sera la bibliothèque de recherche d'usage courant dans la plupart des disciplines) [Arms, 1995]. Les citations qui servent de support et d'illustrations aux figures discutées dans la suite sont donc pour l'essentiel glanées sur le Web. Et l'abondance du corpus est telle que nous nous sommes contenté d'en sélectionner quelques-unes et de les arranger en une sorte de collage qui pourrait presque se passer de commentaire. II va sans dire qu'il a néanmoins fallu recourir aux bibliothèques

\footnotetext{
${ }^{1}$ Son acte de naissance s'établit entre les derniers jours de 1990 et août 1991, d'après l'article "History of the World Wide Web" de Wikipedia: "By Christmas 1990, Berners-Lee had built all the tools necessary for a working Web" (A Noël 1990, Berners-Lee avait bâti tous les outils nécessaires pour un Web opérationnel), et, plus loin, "On August 6 , 1991, Berners-Lee posted a short summary of the World Wide Web project on the alt.hypertext newsgroup. This date also marked the debut of the Web as a publicly available service on the Internet" (Le 6 août 1991, Berners-Lee publia un bref résumé du projet World Wide Web sur la liste alt.hypertext. Cette date marqua aussi le début du Web en tant que service disponible au public).
} 
(universitaires ou personnelle) pour vérifier certaines de ces citations et pour accéder à des textes que les bibliothèques en ligne ne proposent pas - et qui sont souvent les plus fertiles.

\section{Figure 1 : Une métaphore fondatrice}

« présenter une idée sous le signe d'une autre idée plus frappante ou plus connue »

[Fontanier, 1968, p. 99]

Avec cette première figure, on pose une équation Web / bibliothèque qui se présente selon divers degrés: la comparaison, la métaphore in præsentia, la métaphore in absentia, et enfin une curieuse assimilation où la métaphore in præsentia est " réciproque ". Dans cet inventaire, la métaphore in absentia n'est pas la tournure la plus représentée; le plus souvent, il y a coprésence des deux termes. Une explication possible en serait la recherche d'une valeur pédagogique, explicative et heuristique de l'analogie, à travers la mise en évidence de certains traits caractéristiques : "la métaphore ne nomme pas, mais caractérise ce qui est déjà nommé " [Ricœur, 1975, p. 79]. Le mécanisme dans lequel s'inscrit cette équation est bien connu. En voici deux énoncés, qui se rapportent à des contextes tout à fait autres, mais le décrivent parfaitement :

« C'est souvent par approximation que l'on réagit devant un phénomène inconnu : on cherche le morceau de contenu, déjà présent dans notre encyclopédie, qui pourra rendre plus ou moins bien raison du fait nouveau » [Eco, 2009, p. 80].

" L'argument même de l'innovation fait fond sur un imaginaire très ancien, pluriséculaire, de la circulation des objets de savoir. Chacune des nouveautés est immanquablement caractérisée par une image empruntée au temps immémorial des disciplines de la culture » [Jeanneret, 2007].

\section{a. Le Web est comme une bibliothèque}

La comparaison explicite est la forme la plus attendue de l'analogie. Elle permet de faire image sans occulter le caractère d'approximation du rapprochement entre les deux dispositifs. Les deux exemples ci-après ont été sélectionnés en fonction de leur provenance (autorité suprême pour l'une, vox populi pour l'autre), afin de montrer à quel point on a ici affaire à un topos:

"The Web is thus comparable, from the readers' viewpoint, to both a vast library including millions of readily available and indexed publications" (Le Web est donc comparable, du point de vue des lecteurs, à une vaste bibliothèque comportant des millions de publications disponibles et indexées) [Cour Suprême des Etats-Unis, 1997]. 
A la question " A quoi Internet ${ }^{2}$ ressemble le plus ? ", la réponse est à $45 \%$ " une bibliothèque " (contre 15\% pour l'autoroute, et $14 \%$ pour le centre commercial), selon une étude menée aux Etats-Unis en 2000. [Markle Foundation, 2001, p. 24].

Mais on rencontre souvent une forme de comparaison assortie de la mise en exergue d'une différence; le Web n'est pas tout à fait comme une bibliothèque ordinaire, on y trouve des anomalies: "Doing research on the Web is like using a library assembled piecemeal by pack rats and vandalized nightly" (Faire de la recherche sur le Web, c'est comme utiliser une bibliothèque réunie au hasard par des rats des champs et vandalisée toutes les nuits) [Ebert, 1998, p. 66]. Cette figure n'est pas l'apanage des débuts du Web, mais se rencontre de manière persistante: "À I'heure actuelle, Internet ressemble à une immense bibliothèque sans catalogue." [Deschatelets, 2010]. Ce qui conduit à se demander: "why can't the Internet be more like libraries, organised, classified, and with powerful filters in place?" (pourquoi Internet ne peut-il pas être davantage comme les bibliothèques, organisé, classifié et doté de filtres efficaces ?) [Deegan \& Sutherland, 2009, p. 151].

Dans ces correctifs apportés à la comparaison initiale, on voit apparaître le thème de l'organisation de l'information, contrasté avec celui de l'abondance et de la disponibilité qui fonde la comparaison.

\section{b. Le Web est une bibliothèque}

"L'Internet peut être considéré en lui-même comme une gigantesque bibliothèque numérique. " ["Bibliothèque numérique", Wikipédia]. La métaphore in præsentia est très fréquente mais elle est la plupart du temps complétée de qualificatifs ou de modifieurs qui peuvent aller dans le sens d'une mise en avant des qualités manifestées par le Web et seulement potentielles dans une bibliothèque (gigantesque, gratuite, numérique, universelle, etc.) C'est le cas ici dans ce discours du Ministre des Nouvelles technologies de l'information et de la communication (NTIC) de Côte d'Ivoire : "Internet est une bibliothèque gratuite qui vous permet en un temps record d'avoir les informations d'ici et d'ailleurs ॥ [Bakayoko, 2009]; ou encore dans ce texte officiel : "Internet est une bibliothèque planétaire mise à la disposition de tous et plus particulièrement des jeunes. " [Commission nationale consultative des droits de I'homme - France, 2005]. Enfin, selon une étude menée sur 115 étudiants du Département d'anglais de l'Université de Balikesir (Turquie), [Aydin, 2007], 112 d'entre eux (97,4\%) sont d’accord avec la proposition « Internet est une bibliothèque numérique universelle ».

Mais ces adjonctions peuvent également être des correctifs apportés au parallèle dessiné par la métaphore. En écho à la comparaison corrigée dont nous avons fait état ci-dessus, voici une

\footnotetext{
${ }^{2}$ Ici et dans la suite, on tiendra pour acquis l'usage du terme "Internet " pour désigner le Web, au moyen d'une synecdoque généralisante ou d'une métonymie (selon que l'on voie le Web comme un partie d'Internet, ou Internet comme le vecteur du Web).
} 
métaphore (filée) in absentia: "'What we had was a library where all the books were dumped on the floor and there was no card catalogue," Ed Krol, author of "The Hitchhikers Guide to the Internet," said. "Now there's a card catalogue and people are starting to put the books on the shelves."” (" On avait une bibliothèque où tous les livres étaient entassés par terre, et qui n'avait pas de catalogue sur fiches » dit Ed Krol, auteur du Guide d'Internet pour les autostoppeurs. " A présent, il y a un catalogue et les gens commencent à ranger les livres sur les étagères » [Quittner, 1992]. Cette figure se retrouve souvent par la suite avec quelques variantes, mais le plus souvent sous la forme d'une métaphore in præsentia.

Dans une étude menée en 2000, cette métaphore corrigée est résumée ainsi : "The Web was often described as a dysfunctional library" (Le Web a souvent été décrit comme une bibliothèque dysfonctionnelle) [Razan, 2000].

\section{c. Le Web comme bibliothèque}

Un autre ensemble de métaphores de la bibliothèque témoigne d'une opération rhétorique supplémentaire qui fait que la figure initiale n'est plus le focus explicite du discours. Autrement dit, le discours ne pose plus l'analogie du Web avec la bibliothèque mais la présuppose ou la relègue dans l'implicite, c'est-à-dire la fait passer pour allant de soi.

Les deux exemples de substitution qui suivent témoignent de l'intrication conceptuelle que le procédé rhétorique illustre :

"The Internet is the only library needed by somebody doing research in my fields of computer networks and digital libraries" (Internet est la seule bibliothèque dont ait besoin quelqu'un qui fait des recherches dans mes domaines, les réseaux informatiques et les bibliothèques numériques) [Arms, 1995].

"now the paradigm for the universal library is not a library at all, it is the Internet" (A présent le paradigme de la bibliothèque universelle n'est pas une bibliothèque du tout, c'est Internet) [Deegan \& Sutherland, 2009, p. 151].

Comme on le voit, il est parfois malaisé de se représenter la référence pointée par ces énoncés surtout dans le cas du second qui a quelque chose de paradoxal, puisqu'il pose qu'Internet est la bibliothèque universelle, tout en présupposant qu'Internet n'est pas une bibliothèque - et nous ne pensons pas qu'il s'agisse pour autant d'un contre-exemple dans notre corpus de métaphores.

\section{d. ....et réciproquement}

Un dernier exemple (qui pourrait bien être un hapax), mérite d'être cité pour introduire l'idée d'une réversibilité de la métaphore, d'une réciprocité ou d'une assimilation, idée troublante sur le plan (onto)logique, mais tout à fait éclairante pour la lecture des figures qui seront exposées 
dans la suite: "Those who deny that the internet is a library or, conversely, that the library is an internet, are likely to find themselves mistaken" (Ceux qui contestent qu'Internet est une bibliothèque, ou, inversement, que la bibliothèque est un internet, risquent de se trouver dans l'erreur) [Böök, 2010].

\section{Mimèsis : imbrications, mises en abyme et effets miroir}

On nous passera la licence qui consistera dans la suite à utiliser le nom de figures de rhétorique pour caractériser des structures et des phénomènes loin d'être uniquement discursifs. Elle s'autorise d'une volonté d'envisager les pratiques culturelles comme discours et les dispositifs informationnels comme objets signifiants, au-delà des contenus qu'ils véhiculent. Ce qui pourrait constituer notre réponse à la question posée par cette journée d'études : " Le Web a-til un sens? ".

\section{Figure 2 : Métonymies et synecdoques}

« l'existence ou l'idée de l'un se trouvant comprise dans l'existence ou l'idée de l'autre »

[Fontanier, 1968, p. 87]

Entreprendre de classifier les sites, comme l'on fait les répertoires à la Yahoo! dans la première décennie du Web, relève de la transposition vers le nouveau dispositif des technologies intellectuelles d'organisation des connaissances en usage dans l'ancien (classification des livres) [Koch, 1997]; il s'agit d'une pratique mimétique. Mais lorsque l'on indique que ce sont les bibliothécaires qui cataloguent Internet [Callery \& Tracy-Proulx, 1997], que I'on se demande comment le faire ${ }^{3}$, ou lorsque les bibliothèques entreprennent l'archivage du Web ${ }^{4}$, celui-ci y est symboliquement incorporé au même titre que les autres éléments des collections.

De même, l'offre d'accès à Internet en bibliothèque procède d'un traitement banal du Web comme source d'information. Mais lorsque c'est le seul moyen de se connecter, le Web devient une composante de la bibliothèque : "Just over 71 percent of libraries report that they are the only source of free access to computers and the Internet in their communities" (Un peu plus de $71 \%$ des bibliothèques indiquent qu'elles sont la seule source d'accès gratuit aux ordinateurs et à Internet dans leurs communautés) [Davis, Berto \& McClure, 2009].

Le Web est donc dans la bibliothèque. Et la bibliothèque est sur le Web, puis dans le Web. Des catalogues en ligne jusqu'aux bibliothèques numériques actuelles, l'interpénétration des deux types de dispositifs informationnels et les échange de rôles se généralisent en un jeu de miroirs, parfois vertigineux. Avec la mise en ligne précoce des catalogues [Bibliothèque du Congrès,

\footnotetext{
${ }^{3}$ Voir par exemple Journal of Internet Cataloging. Binghamton (NY) : Haworth Press, 1997-2001. [en ligne] http://internetcataloging.com/.

${ }^{4} \mathrm{cf}$. International Internet Preservation Consortium. [en ligne] http://www.netpreserve.org/.
} 
1993], puis le développement et la mise à disposition de fonds numérisés, du projet Gutenberg à Google Books, en passant par Persée et Gallica, le Web devient réceptacle de parties de bibliothèques, puis de bibliothèques entières. Et la bibliothèque peut donc ainsi se cataloguer et s'archiver elle-même...

\section{La bibliothèque universelle}

Dans une première variante de la dialectique morcellement / globalité à travers laquelle se lit cette inclusion mutuelle des deux dispositifs, ce n'est plus Internet qui est vu à présent comme la bibliothèque universelle, mais la réunion des bibliothèques individuelles qui y sont incorporées: "The set of digital libraries on distributed networks can be viewed collectively as a global digital library" (L’ensemble des bibliothèques numériques sur les réseaux distribués peut être vu collectivement comme une bibliothèque numérique mondiale) [Borgman, 2000, p. 151]. C'est là un exemple de décomposition / recomposition entre le genre et ses espèces (le mode $\Sigma$ de [Groupe $\mu, 1982$, p. 100]).

Un autre mode de décomposition / recomposition se fait entre le tout et ses parties (le mode $\Pi$ [ibid.]), par exemple lorsque le Web joue le rôle de catalogue de la bibliothèque, comme dans ce slogan: "Find it on Google, get it from your library" (Trouvez-le sur Google, obtenez-le de votre bibliothèque) [Calhoun, 2006, p. 10]. Adam Hodgkin explicite ce déplacement, lors du lancement de l'initiative de numérisation de Google : "The Google Print vision is of the web as a universal library, with Google Print as an index to that library" (La vision de Google Print est celle du Web comme bibliothèque universelle, avec Google Print comme index de cette bibliothèque) [cité dans The Bookseller du 7 septembre 2005].

Nous ne pourrons guère développer ici le parallèle possible avec une autre révolution informationnelle, mais il faut tout de même citer, à propos de cette vision de la bibliothèque universelle, la remarque d'Erasme sur l'essor de l'imprimerie ${ }^{5}$ : "Aldus bibliothecam molitur, cuius non alia septa sint, quam ipsius orbis" (Alde est en train d'édifier une bibliothèque qui n'a pas d'autres limites que le globe terrestre lui-même) [Erasme, Adages (II.1.1, 17), "Festina lente "]. Et pour clore ces brèves allusions au motif de la bibliothèque universelle, si bien ancré dans notre imaginaire, c'est bien sûr vers Borges que nous nous tournons: "L'univers (que d'autres appellent la Bibliothèque) se compose d'un nombre indéfini, et peut-être infini, [...] ». Et la Bibliothèque de Babel, elle aussi, est en cours de numérisation ${ }^{6}$ : "Chaque livre de la Bibliothèque est constitué de 410 pages, chacune de 40 lignes de 80 signes. [...] Nombre de livres numérisés : 24014 ».

\footnotetext{
${ }^{5}$ C'est le nom de « Google Print » qui incite au parallèle. Il devient « Google Book Search » en 2005, puis c'est l'appellation « Google Books » qui prévaut : parfait exemple de glissement métonymique.

${ }^{6}$ http://dicelog.com/babel.
} 


\section{Un petit détour par Sumatra}

Marco Polo, abordant à Sumatra, est frappé par la faune locale et la décrit : "II ont leofans sauvajes et unicornes aseç que ne sunt mie gueres moin que un leofans: il sunt du po[i]l dou bufal, les pies a fait come leofant, il a un cor enmi la front mout gros et noir. [...] elle est mout laide beste a veoir. II ne sunt pas ensi come nos de ca dion et deviçon: que dient qu'ele se lai<se> prendre a la poucelle, mes vos di qu'il est tout le contraire de celz que nos qui dion que il fust. » (Ils ont des éléphants sauvages, et des licornes, qui ne sont guère moindres : elles ont le poil du buffle et les pieds comme ceux des éléphants, elles ont une corne au milieu du front, très grosse et noire. [...] Ce sont des bêtes bien laides à voir, et elles ne sont pas telles qu'on les décrit chez nous : se laissant prendre dit-on par les pucelles, mais elles sont tout au contraire de ce que nous en disons.) [Polo, 1986, pp. 543-544].

Marco Polo a donc vu des rhinocéros et les a nommés licornes. Umberto Eco, qui a attiré notre attention sur ce passage réjouissant, le commente ainsi : "Marco Polo semble prendre une décision : plutôt que de segmenter encore le contenu en ajoutant un nouvel animal à l'univers des vivants, il corrige la description en vigueur des licornes, qui, si elles existent, sont telles qu'il les a vues, et non telles que la légende le raconte. » [Eco, 2009, p. 82].

C'est à notre sens un mouvement similaire qui conduit à revisiter la bibliothèque-licorne, traditionnelle mais peut-être illusoire, à la lumière des usages et pratiques ayant cours sur le Web-rhinocéros, si présent et massivement réel : "The idea that, in the past ten years, we have so easily accepted the metaphor of the internet as a library has resulted in librarians being displaced from function, and information literacy rendered redundant because a search engine lists hits in a particular way" (L'idée que, dans les dix dernières années, nous avons si facilement accepté la métaphore d'Internet comme bibliothèque a conduit les bibliothécaires à voir leur fonction déplacée et la culture informationnelle à devenir superflue au motif qu'un moteur de recherche liste les résultats d'une certaine manière) [Brabazon, 2008]. Ce qui nous amène à la figure suivante.

\section{Figure 3 : L'imitation}

« consiste à imiter le tour, la construction d'une autre langue »

[Fontanier, 1968, p. 114]

Dns cette figure, la bibliothèque fait comme le Web, ce que l'on pourrait nommer webisme. "The Web is clearly the model for the widespread digital library that Bush (and others) envisaged" (Le Web est clairement le modèle de la bibliothèque numérique extensive que Bush ${ }^{7}$ (et d'autres) envisageait) [Lesk, 1997, p. 25]. Si initialement ce sont les bibliothèques numériques encore balbutiantes que l'on envisage calquées sur le Web, il s'agit à présent

\footnotetext{
${ }^{7}$ Vannevar, pas George...
} 
d'« Adopter l'architecture du Web » [Bermès, 2009, pl. 40] aussi dans les bibliothèques conventionnelles. En mimant le Web (2.0), les bibliothèques 2.0 reproduisent des pratiques informationnelles populaires sur Internet, pour regagner la faveur d'un public que l'on imagine massivement rebuté par les systèmes d'antan. Le Web propose des modèles enviés : "Our users expect simplicity and immediate reward and Amazon, Google, and iTunes are the standards against which we are judged. Our current systems pale beside them" (Nos utilisateurs attendent de la simplicité et une rétribution immédiate, et Amazon, Google et iTunes sont les références à l'aune desquelles nous sommes jugés. Nos systèmes actuels semblent bien pâles en comparaison) [University of California Libraries, 2005, p. 7]. Ce sont l'austérité et la complexité des catalogues, décidément peu en faveur, qui sont visées au premier chef. On parle de "l'ergonomie des outils de référence du web » [Maisonneuve, 2008, p. 5], sur lesquels s'alignent les catalogues: "Pour être bref, l'opac de nouvelle génération se résume en recherche à la Google, avec facettes, nuages de mots et fonctions Web 2.0. » [Maisonneuve, 2008, p. 9].

On a vu plus haut que l'une des métaphores corrigées récurrentes à propos du Web était celle de la bibliothèque sans catalogue. C'est peut-être pour parfaire le mimétisme qu'à l'avenir dans les bibliothèques, les collections entièrement numérisées, indexées automatiquement par les mots de leurs textes, pourront se passer de catalogue: "The catalog is in decline, its processes and structures are unsustainable [...] At the same time, books and serials are not dead, and they are not yet digital. Notwithstanding widespread expansion of digitization projects [...] the role of catalog records in discovery and retrieval of the world's library collections seems likely to continue for at least a couple of decades and probably longer." (Le catalogue est en déclin, ses processus et ses structures ne sont pas viables [...] En même temps, les livres et les périodiques ne sont pas morts, et ne sont pas encore numériques. Malgré l'ample développement des projets de numérisation [...] le rôle des catalogues pour la découverte et le repérage dans les fonds des bibliothèques mondiales devrait perdurer encore une vingtaine d'années et sans doute davantage) [Calhoun, 2006, p. 5].

\section{Figure 4 : Une allégorie?}

« par laquelle on présente une pensée sous l'image d'une autre pensée, propre à la rendre plus sensible et plus frappante »

[Fontanier, 1968, p. 114]

La vision actuelle du Web informationnel passe par un Web de données liées entre elles [Heath \& Bizer, 2011], associées à des métadonnées structurées, standardisées et interprétables : le Web sémantique. II s'agit d'une initiative néguentropique, remède à la bibliothèque en désordre. Cette métaphore était très présente, on l'a vu, aux débuts du Web, et le discours sur le Web sémantique la prolonge volontiers : "Avoir accès à une immense bibliothèque, la plus grande de l'histoire de l'humanité, est une chose extraordinaire, mais qui trouve vite ses limites 
si les informations ne sont pas classées. C'est tout l'enjeu du Web sémantique. Remettre de l'ordre dans les milliards de documents disponibles sur Internet » [SFR Business Team, 2010].

L'architecture du Web sémantique reprend en grande partie le modèle conceptuel général du catalogue de bibliothèque. Les ressources y sont soigneusement décrites par une information secondaire (les métadonnées) elle-même gagée sur l'autorité d’ontologies formalisées; les données ou ressources équivalent aux collections cataloguées, les métadonnées aux notices et les ontologies aux différentes autorités. Et ce modèle de la bibliothèque est souvent revendiqué : "From the W3C's inception, there was a perceived need to bring order to the loosely connected networks of digital documents that made up the Web. Although this order was to be realized by consortium's development of standards, it would also reflect the order that libraries have and the Web does not - a consistent structure by which people can access materials" (Dès les débuts du W3C, on a ressenti le besoin de mettre de l'ordre dans les réseaux faiblement connexes de documents numériques qui ont façonné le Web. Même si cet ordre devait être obtenu à travers le développement de normes par le consortium, il devait aussi refléter l'ordre que les bibliothèques possèdent et pas le Web - une structure cohérente donnant accès aux ressources) [Marshall \& Shipman, 2003]. Ordre, structure et cohérence sont les traits associés à ce modèle, qu'il convient de refléter - ou de réfléchir.

\section{Le principe de réalité}

Au final, on s'interroge sur les raisons pour lesquelles les technologies intellectuelles dévolues à l'organisation de l'information dans les bibliothèques, issues d'une lente maturation et chargées $d^{\prime}$ 'histoire, constituent apparemment le modèle ultime pour une partie des usagers et praticiens du Web, alors que certains professionnels de l'information-documentation semblent aujourd'hui les déconsidérer, et au contraire prôner les technologies les plus récentes issues du Web comme le lieu du souhaitable pour les bibliothèques. Ce qui fonde les analogies que nous venons d'explorer est le caractère d'abondance et de disponibilité des ressources documentaires, caractère commun aux deux dispositifs. Reste que chacun d'entre eux renvoie à l'autre comme ayant accompli ce qu'il n'a pas lui-même atteint: l'accessibilité de l'information pour l'un, l'organisation des connaissances pour l'autre. C'est bien entendu, au moins partiellement, une idéalisation dans les deux cas: "C'est parce que notre esprit nous offre des représentations imaginaires de mondes possibles que nous pouvons demander aux choses d'être de qu'elles ne sont pas. Et ce n'est que lorsqu'elles persistent à être ce qu'elles sont que nous pensons qu'elles nous répondent d'un "non" et qu'elles nous opposent une limite. » [Eco, 2009, p. 78]. 


\section{Références}

\section{(URL visitées le 14/12/2010)}

- ARMS, William Yeo. Digital Library Research in the United States. Follett Lecture Series, 1995. [en ligne] http://www.ukoln.ac.uk/services/papers/follett/arms/paper.html.

- AYDIN, Selami. "Attitudes of EFL learners toward the Internet". The Turkish Online Journal of Educational Technology. vol. 6, n³, 2007, Article 2. [en ligne] http://www.tojet.net/.

- BAKAYOKO, Hamed. Conférence de presse du 22/04/2009. [en ligne] http://www.telecom.gouv.ci/accueil.php?page=presse\&presse=lapresse\&id=18.

- BENTLEY, Paul. "Talking up the back end in an evolving revolution: the VALA Conference 2010". Online Currents. Juin 2010. [en ligne] http://www.twf.org.au/research/Talkingupbackend.html.

- BERMES, Emmanuelle. "Les catalogues de bibliothèques sur le Web " (41 Diapositives) in : Journée d'études MEDIAL : les catalogues nouvelle génération. Bibliothèque nationale de France, 8 juin 2009. [en ligne] http://www.slideshare.net/Figoblog/les-catalogues-sur-le-web.

- BIBLIOTHĖQUE DU CONGRÈS. "Gaining Electronic Access to the World's Largest Library". Library of Congress Information Bulletin. vol. 52, n¹6, septembre 1993. [en ligne] http://www.loc.gov/loc/lcib/93/9316/infosys.html.

- "Bibliothèque numérique." Wikipédia, I'encyclopédie libre. 15 mars 2011. [en ligne] http://fr.wikipedia.org/w/index.php?title=Biblioth\%C3\%A8que num\%C3\%A9rique\&oldid=63235136.

- BÖÖK, Mikael. "Editorial". Information for Social Change. 2010. [en ligne] http://www.libr.org/isc/issues/ISC30/issue30.pdf.

- BORGES, Jorge Luis. "La Bibliothèque de Babel » in : CEuvres complètes. Tome I. Paris : Gallimard, 1993. (Bibliothèque de la Pléiade, $n^{\circ}$ 400). pp. 491-498.

- BORGMAN, Christine L. From Gutenberg to the Global Information Infrastructure: Access to Information in the Networked World. Cambridge (Mass.) : MIT Press, 2000.

- BRABAZON, Tara. "Come back Zenodotus. All is forgiven". Times Higher Education. 10 avril 2008. [en ligne] http://www.timeshighereducation.co.uk/story.asp?storyCode $=401438$.

- BURNETT, Robert \& MARSHALL, David. Web theory: an introduction. Londres : Routledge, 2003.

- CALHOUN, Karen. The Changing Nature of the Catalog and its Integration with Other Discovery Tools; Final Report, Prepared for the Library of Congress. 2006. [en ligne] http://www.loc.gov/catdir/calhounreport-final.pdf.

- CALLERY, Anne \& TRACY-PROULX, Deb."Yahoo! Cataloging the Web". Journal of Internet Cataloging. vol. 1, n 1, 1997. pp. 57-64. [en ligne] http://www.library.ucsb.edu/untangle/callery.html.

- CASEY, Michael E. \& SAVASTINUK, Laura C. "Library 2.0: Service for the Next-Generation Library." Library Journal. vol. 131, n¹4, 2006. pp. 40-42.

- COMMISSION NATIONALE CONSULTATIVE DES DROITS DE L'HOMME, FRANCE. Prix René Cassin des établissements scolaires 2005-2006. [en ligne] http://www.cncdh.fr/archives_prix.php3?id_article=84.

- COUR SUPREME DES ETATS-UNIS. Reno vs ACLU (1997), cité par KOEHLER, Wallace. "Digital libraries and World Wide Web sites and page persistence". Information Research. Vol. 4 n4, juillet 1999. [en ligne] http://informationr.net/ir/4-4/paper60.html.

- DAVIS, D. M. , BERTOT, J. C. \& MCCLURE, C. R. Libraries Connect Communities 3: Public Library Funding \& Technology Access Study. Chicago : American Library Association, 2009. [en ligne] http://www.ii.fsu.edu/plinternet.

- DESCHATELETS, Jacinthe. Dossier sur les métadonnées. Association internationale francophone des bibliothécaires et documentalistes (AIFBD) : 2010. [en ligne] http://www.aifbd.org/index.php?option=com content\&view=article\&id=129\&/temid=96

- DEEGAN, Marilyn \& SUTHERLAND, Kathryn. Transferred illusions: digital technology and the forms of print. Farham (Royaume-Uni) : Ashgate, 2009. 
- EBERT, Roger. "Critical Eye". Yahoo! Internet Life. Septembre 1998.

- ECO, Umberto. Kant et l'ornithorynque. (trad. Julien Gayrard). Paris : Librairie générale française, 2009. (1ère éd. Paris : Grasset, 2001). (Le livre de poche).

- FONTANIER, Pierre. Les figures du discours. Paris : Flammarion, 1968.

- GROUPE $\mu$. Rhétorique générale. Paris : Seuil, 1982. (Points).

- HEATH, Tom \& BIZER, Christian. Linked Data: Evolving the Web into a Global Data Space. San Rafael (CA) : Morgan \& Claypool, 2011. [en ligne] http://linkeddatabook.com/book.

- "History of the World Wide Web". Wikipedia : The free Encyclopedia. 15 mars 2011. [en ligne] http://en.wikipedia.org/w/index.php?title=History of the World Wide Web\&oldid=417922219.

- JEANNERET, Yves. "Comment faire, que faire, pourquoi faire ? Non-synthèse " in Actes des Journées d'études " Sites Internet: quelles ressources pour les visiteurs ? " Paris, Palais de la Découverte, 21 et 22 novembre 2007. [en ligne] http://www.ocim.fr/Actes-des-journees-d-etudes-Sites.html

- $\mathrm{KOCH}$, Traugott. Specification for resource description methods Part 3. The role of classification schemes in Internet resource description and discovery. 1997. [en ligne] http://www.ukoln.ac.uk/metadata/desire/classification/classification.pdf

- LESK, Michael. Practical digital libraries: books, bytes, and bucks. San Francisco: Morgan Kaufmann, 1997.

- MAISONNEUVE, Marc. Le catalogue de la bibliothèque à l'heure du Web 2.0 : étude des opacs de nouvelle génération. Paris : ADBS, 2008.

- MARKLE FOUNDATION. Toward a Framework for Internet Accountability. 2001. [en ligne] http://www.markle.org/markle programs/project archives/2000/frameworkaccountability.php.

- MARSHALL, C.C. \& SHIPMAN, F.M. "Which semantic web?" in : HYPERTEXT'03: Proceedings of the fourteenth ACM conference on Hypertext and hypermedia. New York : ACM Press, 2003. pp 57-66.

- POLO, Marco. Milione - Le divisament dou monde. Milan : Arnoldo Mondadori, 1986. (3 ${ }^{\text {ème }}$ éd.).

- QUITTNER, Joshua. "Getting up to speed on the computer highway". Newsday. 3 novembre 1992. pp. 51, 54-56.

- RATZAN, Lee. "Making sense of the Web: a metaphorical approach". Information Research. vol. 6, n¹, 2000. [en ligne] http://InformationR.net/ir/6-1/paper85.html.

- RICCEUR, Paul. La métaphore vive. Paris : Seuil, 1975. (L’Ordre philosophique).

- SFR BUSINESS TEAM. Le Web sémantique, ou l'art de cataloguer Internet. 10 mars 2010. [en ligne] http://www.sfrbusinessteam.fr/faire-equipe-avec-vous/actualite-sfr-business-team/nouveauxusages/web-semantique.jsp.

- UNIVERSITY OF CALIFORNIA LIBRARIES. Rethinking How We Provide Bibliographic Services for the University of California: Final Report, December 2005. 2005. [en ligne]

http://libraries.universityofcalifornia.edu/sopag/BSTF/Final.pdf. 